\title{
Effects of Acoustic Stimuli on Neuronal Activity in the Auditory Cortex of the Rat
}

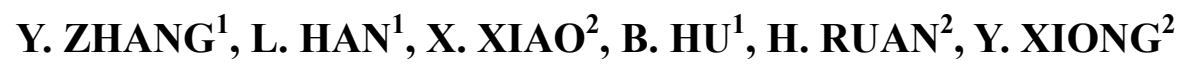 \\ ${ }^{1}$ Department of Physiology, Third Military Medical University, Chongqing, P. R. China, \\ ${ }^{2}$ Department of Neurobiology, Third Military Medical University, Chongqing, P. R. China
}

Received September 18, 2010

Accepted March 1, 2011

On-line May 16, 2011

\begin{abstract}
Summary
Spontaneous activity of cortical neurons exhibits alternative fluctuations of membrane potential consisting of phased depolarization called "up-state" and persistent hyperpolarization called "down-state" during slow wave sleep and anesthesia. Here, we examined the effects of sound stimuli (noise bursts) on neuronal activity by intracellular recording in vivo from the rat auditory cortex (AC). Noise bursts increased the average time in the up-state by $0.81 \pm 0.65 \mathrm{~s}$ (range, $0.27-1.74 \mathrm{~s}$ ) related to a $10 \mathrm{~s}$ recording duration. The rise times of the spontaneous

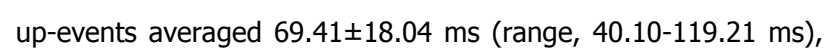
while those of the sound-evoked up-events were significantly shorter $(p<0.001)$ averaging only $22.54 \pm 8.81 \mathrm{~ms}$ (range, 9.31$45.74 \mathrm{~ms}$ ). Sound stimulation did not influence ongoing spontaneous up-events. Our data suggest that a sound stimulus does not interfere with ongoing spontaneous neuronal activity in auditory cortex but can evoke new depolarizations in addition to the spontaneous ones.
\end{abstract}

\section{Key words}

Auditory cortex - Intracellular recording - Depolarization • Spontaneous activity $\bullet$ Rhythmic discharges

\section{Corresponding author}

Y. Xiong, Department of Neurobiology, Third Military Medical University, Chongqing, P. R. China. Fax: 86-23-68752232. E-mail: xiongying2001@yahoo.com

\section{Introduction}

Spontaneous activity of the neocortex, including the sensory cortices, appears as periodic burst firing of single units and periodic negative waves of local field potentials, and is also reflected in a slow oscillation of the electroencephalogram (EEG) during sleep and anesthesia (Eggermont et al. 1993, Amzica and Steriade 1995). The cellular basis of rhythmic cortical events is found in fluctuations of the membrane potential of cortical neurons consisting of short pulsed depolarizations called "upstate" and hyperpolarizations of longer duration called "down-state" (Amzica and Steriade 2002). These rhythmic events are observed not only in cortical neurons in vivo, but also in cortical slices in vitro in which the thalamocortical inputs are absent (Sanchez-Vives and McCormick 2000, Mao et al. 2001, Ikegaya et al. 2004). This suggests that spontaneous activity originates from local cortical circuits.

In the sensory cortex, prolonged visual stimulation can increase the probability of the up-state in complex neurons of the visual cortex (Anderson et al. 2000). Whisker-evoked responses in the somatosensory cortex are dependent on the membrane state (i.e. up- or down-state) (Sachdev et al. 2004). In the auditory cortex (AC), previous experiments showed spontaneous membrane potential fluctuations and their ionic mechanism (Metherate and Ashe 1993). Compared with studies on other cortices (i.e. somatosensory and visual cortices), little is known about the effects of acoustic stimuli on the membrane potential fluctuations of the AC neurons. The aim of the present study was to investigate the effects of acoustic stimuli on spontaneous membrane potential fluctuations in the rat $\mathrm{AC}$ using in vivo intracellular recording. We found that acoustic stimuli can evoke up-events during the spontaneous hyperpolarization phase (down-state), but have no effect on the membrane potential during the depolarization 
phase (up-state). In addition, the level of resting membrane potential appeared to be a major factor that determined the amplitudes of both spontaneous and sound-evoked up-events.

\section{Methods}

Experiments were carried out in 45 female Sprague-Dawley rats with body weight ranging from 200 to $250 \mathrm{~g}$. All protocols and procedures were in accordance with Ethics in the Care and Use of Laboratory Animals of China and approved by the Animal Care and Use Committee of the Third Military Medical University.

\section{Animal preparation}

All surgeries and experiments were performed under anesthesia by intraperitoneal injection of urethane (ethyl carbamate, $1.5 \mathrm{~g} / \mathrm{kg}$ ). An additional dosage of urethane $(0.2 \mathrm{~g} / \mathrm{kg})$ was given when rats showed responses to tail pinch. Tracheotomy was performed and the trachea was cannulated in order to maintain smooth breathing during experiments. Then the animal's head was immobilized with a custom-made head clamp by rigidly clamping between the palate and nasal/frontal bones. The head clamp was adjusted to align bregma and lambda points of the skull in one horizontal plane. The rat's body was suspended by hanging up the caudal back. In order to minimize the pressure difference between the body cavities and the skull cavity, the body level usually was adjusted $15-30^{\circ}$ higher than the horizontal level (Konopacki et al. 2003). By doing this, cortical fluctuation caused by breathing can largely be eliminated. The scalp was incised along the midline and subcutaneous tissue and muscle were removed to expose the right skull. AC of the rat typically lies at the dorsolateral portion of the temporal cortex and is framed by a characteristic blood vessel pattern (Kelly and Sally 1988). A large area of the skull (about $3 \mathrm{~mm} \times 3 \mathrm{~mm}$ ) was thinned above this region using a reliable dental device (Drill: Strong 90, Saeshin Precision Co. Ltd, Korea; Bur: SSW HP-2, SS White Burs, Inc., Lakewood, NJ, USA) such that a thin, well-polished, transparent, bone membrane remained (Pinault 2005). This procedure helped to easily identify the blood vessel pattern on the temporal cortex and to avoid blood vessels when a hole was opened. A hole of less than $1 \mathrm{~mm}$ in diameter was made roughly at the center of this area. The dura was gently incised using a sharp needle mounted on a 1-ml syringe and ending in a miniature hook (made when gently scratching a piece of metal with the tip of the needle). This small-hole surgical technique avoids brain movements by keeping the brain's volume constant within the cranial cavity and does not require additional technical procedures (Pinault 2005). The exposed cortex was kept moist and protected with warm paraffin oil. The surgery and subsequent electrophysiological experiments were performed in an electrically shielded and anechoic chamber. The body temperature of the rat was maintained at $37^{\circ} \mathrm{C}$ by using a U-shape feedback-controlled heating pad that was placed just beneath the rat's body.

\section{Acoustic stimulus}

White noise or tone bursts of $60 \mathrm{~ms}$ in duration and $5 \mathrm{~ms}$ in rise/decay time were used to evoke auditory responses in cortical neurons. Acoustic signals were digitally synthesized and converted to analog signals by a Real-time Processor (RP2, Tucker-Davis Technologies, Alachua, FL, USA). The output amplitude of the sinusoidal waves from the RP2 was set at $20 \mathrm{~V}$ peak-topeaks. The signals were then fed to an attenuator (PA5) and presented by an electrostatic speaker (ES1) via an electrostatic speaker driver (ED1). The speaker was placed $45^{\circ}$ to the left of and $10 \mathrm{~cm}$ away from rat's left ear. During the experiment, acoustic stimuli were played by BrainWare data acquisition software (Tucker-Davis Technologies). This software also allowed setting the frequency of tone bursts and the attenuation of the PA5. The noise or tone amplitude was expressed as decibel sound pressure level (dB SPL, ref. $20 \mu \mathrm{Pa}$ ). The output of the electrostatic speaker was calibrated at the position of the animal's left ear with a condenser microphone (Model 377A01, PCB Piezotronics, NY, USA) and a microphone preamplifier (Model 426B03, PCB Piezotronics). The signal for calibration was not attenuated. Frequency and amplitude of tone bursts were varied manually with BrainWare software.

Recording of spontaneous and tone-evoked activities in the primary auditory cortex

A tungsten electrode of $\sim 2 \mathrm{M} \Omega$ tip impedance was first used for extracellular recording, to determine the best frequency and minimum threshold of cortical neurons. The electrode was advanced perpendicularly to the surface of the AC.

Tone-evoked responses were commonly observed when the electrode tip was $\sim 500 \mu \mathrm{m}$ below the cortical surface. Electrical signals were filtered with a bandpass of $0.3-10 \mathrm{kHz}$ and amplified 10,000 times with 
a RA16 module (Tucker-Davis Technologies). The output signals were monitored on an oscilloscope. A noise burst was delivered at a rate of 1 per second during electrode penetration. Once noise-evoked responses were observed, the best frequency and minimum threshold of the recorded neurons were measured with manual variation of the tone frequency and amplitude. The tungsten electrode was then removed and a sharp glass-pipette filled with $1.0 \mathrm{M}$ potassium acetate was employed for intracellular recording. The tip impedance of the sharp glass electrode ranged between 65 and $90 \mathrm{M} \Omega$ (Konopacki et al. 2003). The sharp glass electrode was placed at a location adjacent to the penetrating point made by the tungsten electrode and penetrated perpendicularly to the AC surface by a stepping motor at a step width of 1 or $2 \mu \mathrm{m}$.

\section{Data acquisition and analysis}

Upon penetrating the membrane of a cell, the electrode detected a sharp drop of membrane potential. Neurons showing a resting membrane potential less than $50 \mathrm{mV}$ commonly indicated unhealthy cells and were excluded from the present study. Electrical signals were first fed to a wide-band active probe electrometer (intra 767, World Precision Instruments, Sarasota, FL, USA), and then stored on a computer via an analog-to-digital converter (Digidata 1332A; Axon Instruments, Foster City, CA). Data were analyzed off-line using pClamp 10.0 software (Axon Instruments).

Data are expressed as mean \pm standard deviation (S.D.). Student's $t$-test was used to examine the significance between two sets of data, using $95 \%$ as the confidence level $(\mathrm{p}<0.05)$.

\section{Results}

We successfully recorded stable activities of 50 neurons sampled from the primary auditory cortices of 45 animals. The depths from which neurons were sampled ranged from $256 \mu \mathrm{m}$ to $815 \mu \mathrm{m}$ below the brain surface. Neuronal best frequencies and minimum thresholds measured with extracellular recording ranged from $5.4 \mathrm{kHz}$ to $34.6 \mathrm{kHz}$ and from $7.8 \mathrm{~dB}$ SPL to $41.2 \mathrm{~dB}$ SPL respectively (average 17.7 $\pm 8.6 \mathrm{~dB}$ SPL).

All sampled neurons exhibited spontaneous alteration of membrane potential, on average, switching between down-state $(-72.4 \pm 6.9 \mathrm{mV})$ and up-state $(-58.6 \pm 4.6 \mathrm{mV})$ (Fig. 1A). The spontaneous switching of the membrane potential occurred at a rate of
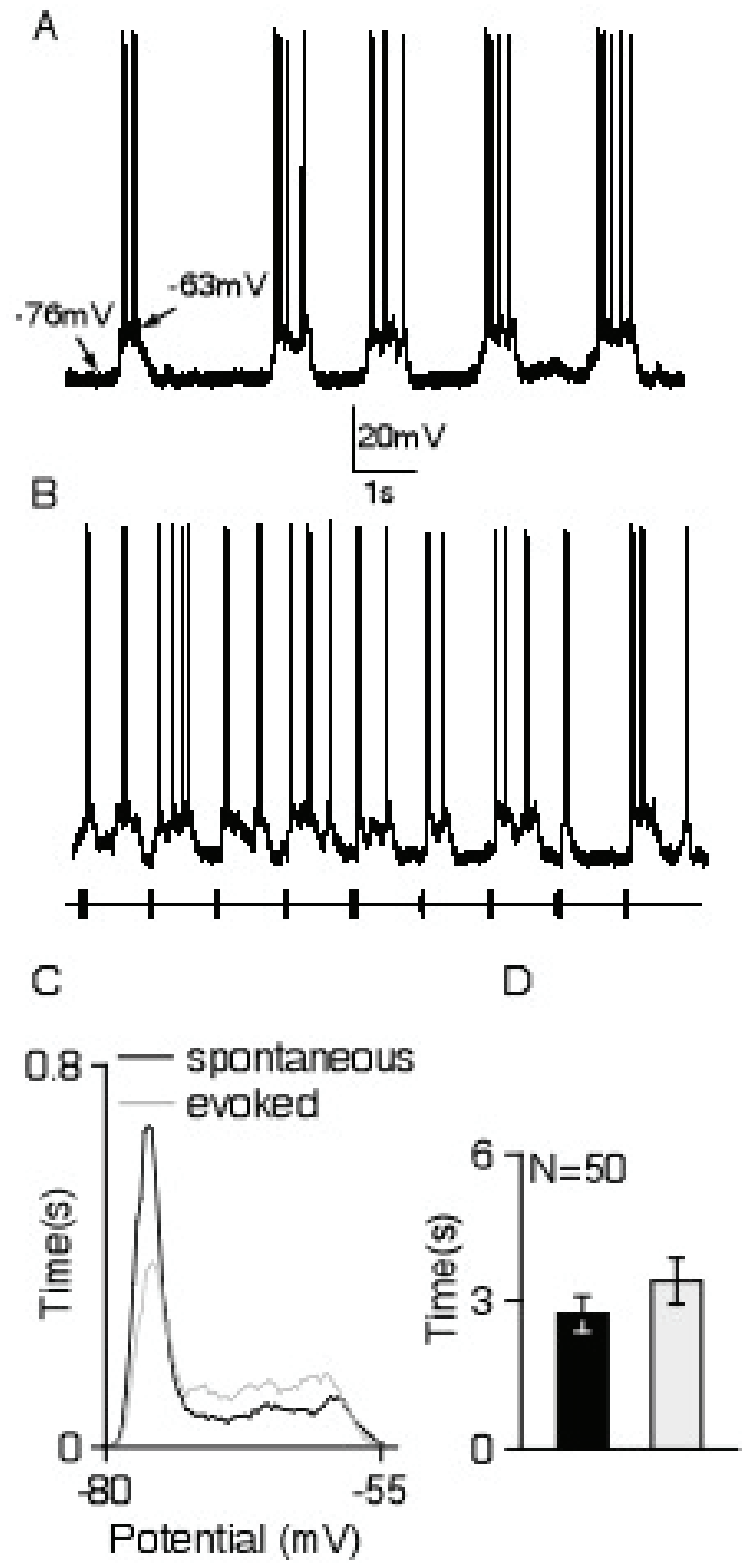

Fig. 1. Spontaneous (A) and noise-evoked activity (B) of the same $A C$ neuron. In $B$, the timing of the noise bursts is indicated on the trace below the recording. C. All-points histogram showing the amount of time spent at any given membrane potential for the recordings shown at A (black) and B (gray). Histograms do not include the action potentials. D. Average time from a $10 \mathrm{~s}$ measuring period of membrane potentials from all 50 neurons in an up-state due to spontaneous (black) or soundevoked (gray) conditions.

$0.54 \pm 0.36 \mathrm{~Hz}$. The duration of spontaneous up-events lasted for an average of $0.64 \pm 0.32 \mathrm{~s}(\mathrm{n}=50$ cells). Sound evoked up-events occurred when a noise burst with $50 \mathrm{~dB}$ SPL was presented during the spontaneous down-state (Fig. 1B, Fig. 3); the duration of noise-evoked up- events lasted for an average of $0.38 \pm 0.21 \mathrm{~s}(\mathrm{n}=50$ cells). To better demonstrate the effects of the acoustic stimuli on spontaneous activity, the membrane potentials at each 

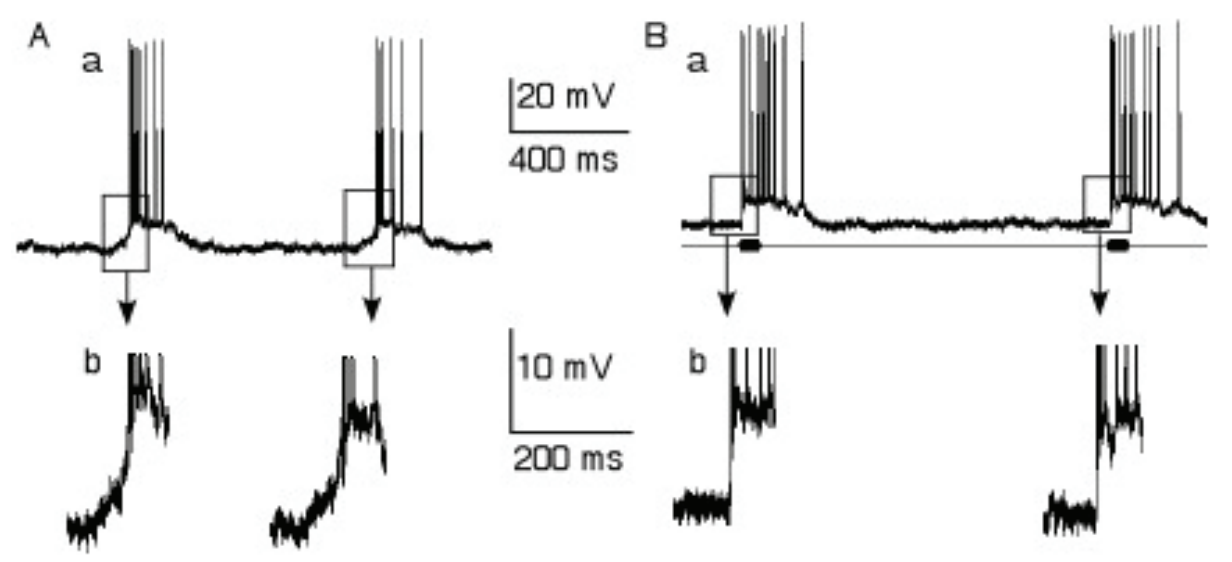

Fig. 2. Rise time of spontaneous and noise-evoked up-events. A. Typical recording of spontaneous up events $(A a)$, which is shown time-expanded by a factor of two in the lower panel (Ab). B. Same as in A, but for noise-evoked up events.

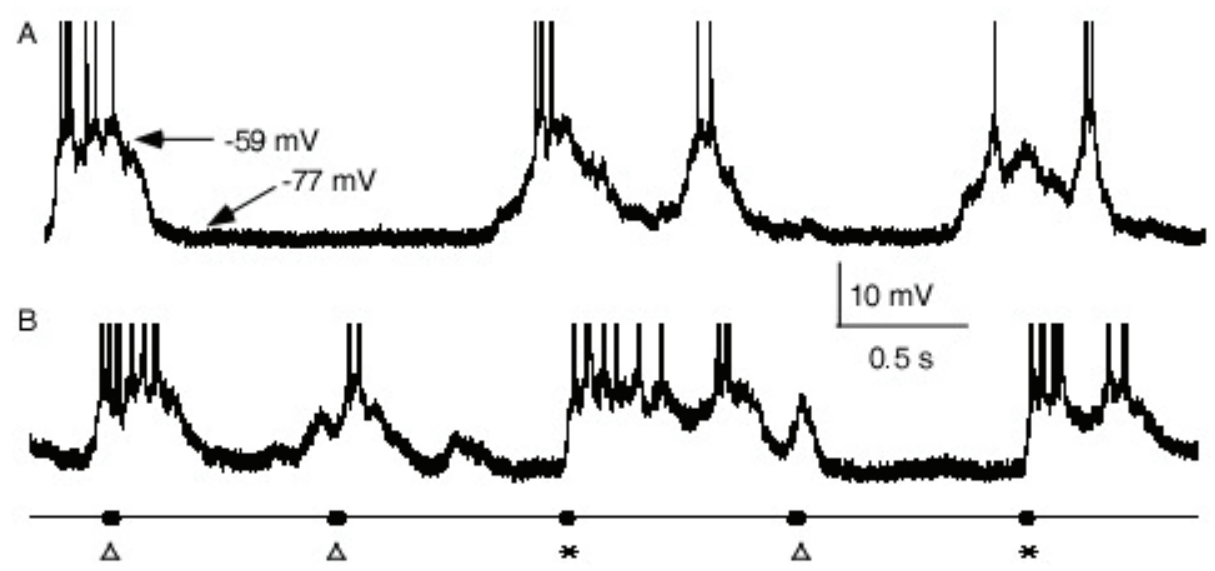

Fig. 3. A. Typical recording of spontaneous activity from an AC neuron. B. Noise bursts have no effect on the neuron during the upstate (indicated by triangles under the stimulus trace), but can evoke responses during the down-state (indicated by asterisks under the stimulus trace). Spikes are truncated in both recordings.

time point are presented as frequency histograms from the $10 \mathrm{~s}$ recording segments shown in Figure $1 \mathrm{~A}$ and $\mathrm{B}$ (action potentials excluded). These will be called allpoint histograms, which is consistent with those used in analyzing data from single-channel recordings (Stern et al. 1997). The result is shown in Figure 1C for spontaneous- (black line) and sound-evoked activity (gray line). The histograms from both spontaneous and noise-evoked activity showed clear differences in the durations at the resting potential levels, with a smaller peak (shorter duration) in the noise-evoked case. In addition, membrane potentials above the resting potential occurred generally for longer durations in the noiseevoked case compared to the spontaneous case. This is quantified for $10 \mathrm{~s}$ recording segments from all 50 neurons in Figure 1D, which shows that the average time spent in the up-state mode increased by $0.81 \pm 0.65 \mathrm{~s}$ (range, 0.27-1.74 s) from the spontaneous to the noiseevoked case.

Figure 2 shows typical recordings of spontaneous (Fig. 2A) and noise-evoked up-events (Fig. $2 \mathrm{~B})$. The rise phases of the up-events are shown timeexpanded by a factor of two in Figure $2 \mathrm{Ab}$ and $2 \mathrm{Bb}$. The rise times for the spontaneous events ranged from 40.10 to $119.21 \mathrm{~ms}$, averaging $69.41 \pm 18.04 \mathrm{~ms}$. The rise times for the sound-evoked events were significantly shorter $(\mathrm{p}<0.001)$. They ranged from 9.31 to $45.74 \mathrm{~ms}$, averaging $22.54 \pm 8.81 \mathrm{~ms}$.

Since a sound stimulus can increase the time spent in the up-state (Fig. 1D), we wanted to know how the sound stimulus affected the spontaneous up-state. We compared two cases: noise bursts occurring coincidently with spontaneous up-states or in intervals between spontaneous up-states. Figure 3A shows a typical recording of spontaneous fluctuations of the membrane potential of an AC neuron. When the noise bursts were repeatedly presented at a rate of $1 / \mathrm{s}$, they evoked upevents during the down-state of the membrane potential, but had no effect on the membrane potential during the spontaneous up-state (Fig. 3B).

Finally, we found that the relative amplitudes between the actual resting potentials and the membrane potentials of the up-states were related to the level of the resting potentials in the same way for both the spontaneous and the sound-evoked up-states. The correlations with regression lines are shown in Figure 4A for spontaneous $(\mathrm{r}=0.79, \mathrm{p}<0.001 ; \mathrm{n}=50)$ and in Figure $4 \mathrm{~B}$ for noise-evoked $(\mathrm{r}=0.74, \mathrm{p}<0.001 ; \mathrm{n}=50)$ up-states. 
This result indicates that the cortical neurons show the same average amount of depolarization spontaneously and in response to noise bursts, the magnitude of the depolarization depending only on the level of the resting potential.

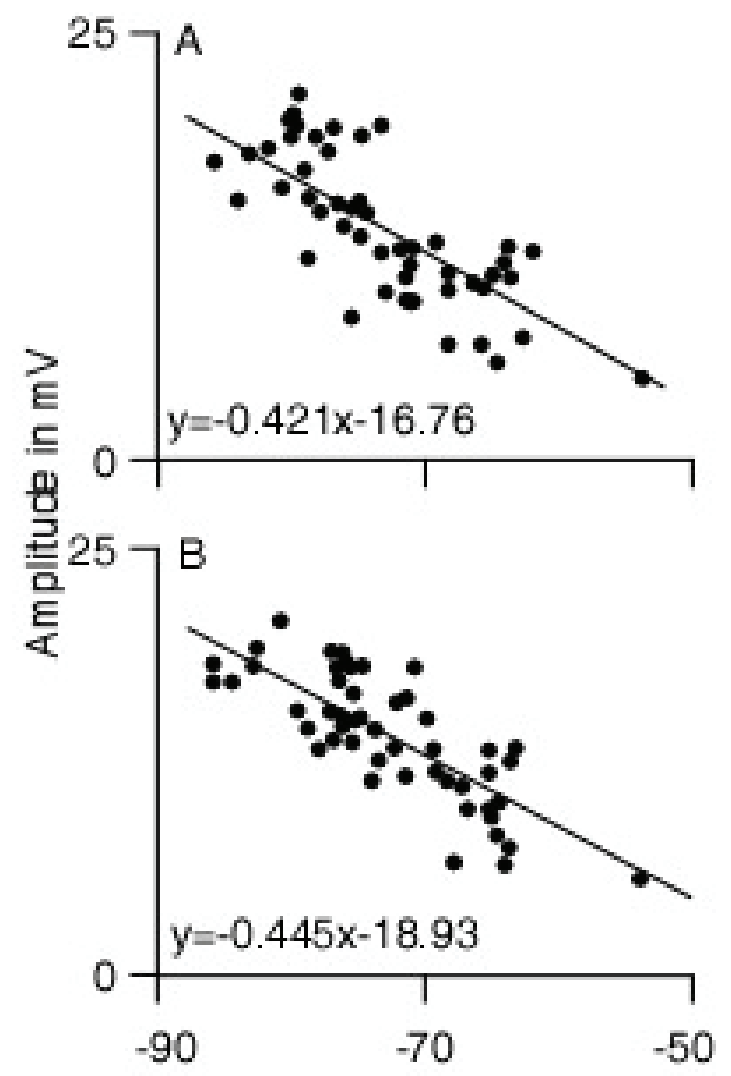

\section{Resting membrane potential in $\mathrm{mV}$}

Fig. 4. The relative amplitude of both spontaneous (A) and sound-evoked (B) up events ( $y$-axis) varies linearly with the value of the resting membrane potential (x-axis). Equations of the respective regression lines are indicated.

\section{Discussion}

Although there is clear diversity in the architecture and function of different cortical areas such as the prefrontal cortex and sensory cortices, spontaneous activity is commonly observed in quiescence and appears in similar fashion (Eggermont et al. 1993, Amzica and Steriade 1995). In natural sleep or under anesthesia, spontaneous discharges typically occur with temporal regularity. Specifically, this temporal regularity is periodic, showing up in rhythmic spike bursts of single units, negative waves of local field potentials, and slow oscillations of local EEG waves at a rate of less than $1 \mathrm{~Hz}$ (Steriade et al. 1993). Both in vivo and in vitro studies have revealed that the spontaneous activity of the cortex is based on the cyclic switching of the activity state of cortical networks (Mao et al. 2001, Shu et al. 2003, Ikegaya et al. 2004). In agreement with previous studies in other sensory cortices, our data show that periodic switching of membrane potentials between two states was found in all sampled AC neurons at a rate of $0.54 \pm 0.36$ $\mathrm{Hz}$, without exception. In most cases, depolarization of the neuronal membrane potential resulted in burst firing. Such low-frequency spontaneous activity of the cortex may be generated in and propagated over cortical intrinsic circuits, although the exact location of the generator is as yet unknown (Steriade et al. 1993, Sanchez-Vives and McCormick 2000).

Our results indicate that an acoustic stimulus can induce up-state events during time intervals of spontaneous down-states, leading to an overall increased time of up-state. This increase was not due to the increase of the duration of single up-events but rather to the increase of the number of the up-events induced by the sound stimuli. Since bistable fluctuations of the membrane potential arise largely from synaptic activity (Stern et al. 1997, 1998, Stevens and Zador 1998), sensory stimulation may not act independently on individual cortical cells, but seems to interact at the network level with the mechanism generating synchronized fluctuations of membrane potentials within a neuronal populations (Anderson et al. 2000).

For individual cortical neurons, excitatory synapses arising from intracortical connections are greater in number than synapses from thalamocortical projections (Ahmed et al. 1994, Douglas et al. 1995) whereas the latter are greater in strength (Stratford et al. 1996, Gil et al. 1999). This suggests that the activity of individual cortical neurons could potentially be impacted in different ways by activities of both intracortical and thalamocortical fibers. In our study, the rise time was significantly shorter in sound-evoked up-events. This difference could result from stronger thalamocortical compared to spontaneous intracortical synaptic activity.

It is noticeable that acoustic stimulation induced depolarization of cortical neurons only when delivered in between the spontaneous up-states (Fig. 3). These findings are in general agreement with previous reports from the somatosensory cortex in vivo (Petersen et al. 2003) and the thalamocortical brain slice (Watson et al. 2008). These studies demonstrated that sensory or electrical stimulation of the thalamus did not alter ongoing cortical activity during the up-state. This 
indicates that neural computation or integration of sensory information shares similar mechanisms across different sensory cortices. The underlying mechanism may be related to the increased membrane conductance during the up-state when membrane potentials reach the firing-threshold level.

Our data showed that the amplitudes of both spontaneous and sound-evoked up-events were related linearly to the levels of resting membrane potentials: the lower the resting membrane potential, the larger the depolarization amplitude (Fig. 4). This phenomenon was also reported in previous studies both in vivo and in vitro (Thomson 1986, Amzica and Steriade 1995, Petersen et al. 2003) and was regarded as being dependent on
NMDA-mediated depolarization (Thomson 1986).

\section{Conflict of Interest}

There is no conflict of interest.

\section{Acknowledgements}

We thank Dr Jun Yan (University of Calgary) for his assistance in data processing and suggestions on writing of the manuscript. This work was supported by grants of the National Natural Foundation of China (No.30770681, 30911120490, 31070970) and Chongqing (2008BB520) and Scientific Research Foundation for Returned Scholars, Ministry of Education of China.

\section{References}

ANDERSON J, LAMPL I, REICHOVA I, CARANDINI M, FERSTER D: Stimulus dependence of two-state fluctuations of membrane potential in cat visual cortex. Nat Neurosci 3: 617-621, 2000.

AHMED B, ANDERSON JC, DOUGLAS RJ, MARTIN KA, NELSON JC: Polyneuronal innervation of spiny stellate neurons in cat visual cortex. J Comp Neurol 341: 39-49, 1994.

AMZICA F, STERIADE M: Short- and long-range neuronal synchronization of the slow $(<1 \mathrm{~Hz})$ cortical oscillation. J Neurophysiol 75: 20-38, 1995.

AMZICA F, STERIADE M: The functional significance of K-complexes. Sleep Med Rev 6: 139-149, 2002.

DOUGLAS RJ, KOCH C, MAHOWALD M, MARTIN KA, SUAREZ HH: Recurrent excitation in neocortical circuits. Science 269: 981-985, 1995.

EGGERMONT JJ, SMITH GM, BOWMAN D: Spontaneous burst firing in cat primary auditory cortex: age and depth dependence and its effect on neural interaction measures. J Neurophysiol 69: 1292-1313, 1993.

GIL Z, CONNORS BW, AMITAI Y: Efficacy of thalamocortical and intracortical synaptic connections: quanta, innervation, and reliability. Neuron 23: 385-397, 1999.

IKEGAYA Y, ARON G, COSSART R, ARONOV D, LAMPL I, FERSTER D, YUSTE R: Synfire chains and cortical songs: temporal modules of cortical activity. Science 304: 559-564, 2004.

KELLY JB, SALLY SL: Organization of auditory cortex in the albino rat: sound frequency. J Neurophysiol 59: 1756$1769,1988$.

KONOPACKI J, BLAND BH, DYCK R: Intracellular recording and labeling of neurons in midline structures of the rat brain in vivo using sharp electrodes. J Neurosci Methods 127: 85-93, 2003.

MAO BQ, HAMZEI-SICHANI F, ARONOV D, FROEMKE RC, YUSTE R: Dynamics of spontaneous activity in neocortical slices. Neuron 32: 883-898, 2001.

METHERATE R, ASHE JH: Ionic flux contributions to neocortical slow waves and nucleus basalis-mediated activation: whole-cell recordings in vivo. J Neurosci 13: 5312-5323, 1993.

PETERSEN CC, HAHN TT, MEHTA M, GRINVALD A, SAKMANN B: Interaction of sensory responses with spontaneous depolarization in layer 2-3 barrel cortex. Proc Natl Acad Sci U S A 100: 13638-13643, 2003.

PINAULT D: A new stabilizing craniotomy-duratomy technique for single-cell anatomo-electrophysiological exploration of living intact brain networks. J Neurosci Methods 141: 231-242, 2005.

SACHDEV RN, EBNER FF, WILSON CJ: Effect of subthreshold up and down states on the whisker-evoked response in somatosensory cortex. $J$ Neurophysiol 92: 3511-3521, 2004.

SANCHEZ-VIVES MV, MCCORMICK DA: Cellular and network mechanisms of rhythmic recurrent activity in neocortex. Nat Neurosci 3: 1027-1034, 2000. 
SHU Y, HASENSTAUB A, MCCORMICK DA: Turning on and off recurrent balanced cortical activity. Nature 423: 288-293, 2003.

STERIADE M, NUÑEZ A, AMZICA F: A novel slow $(<1 \mathrm{~Hz})$ oscillation of neocortical neurons in vivo: depolarizing and hyperpolarizing components. J Neurosci 13: 3252-3265, 1993.

STERN EA, KINCAID AE, WILSON CJ: Spontaneous subthreshold membrane potential fluctuations and action potential variability of rat corticostriatal and striatal neurons in vivo. J Neurophysiol 77: 1697-1715, 1997.

STERN EA, JAEGER D, WILSON CJ: Membrane potential synchrony of simultaneously recorded striatal spiny neurons in vivo. Nature 394: 475-478, 1998.

STEVENS CF, ZADOR AM: Input synchrony and the irregular firing of cortical neurons. Nat Neurosci 1: 210-217, 1998.

STRATFORD KJ, TARCZY-HORNOCH K, MARTIN KA, BANNISTER NJ, JACK JJ: Excitatory synaptic inputs to spiny stellate cells in cat visual cortex. Nature 382: 258-261, 1996.

THOMSON AM: A magnesium-sensitive post-synaptic potential in rat cerebral cortex resembles neuronal responses to N-methylaspartate. J Physiol Lond 370: 531-549, 1986.

WATSON BO, MACLEAN JN, YUSTE R: Up states protect ongoing cortical activity from thalamic inputs. PLoS One 3: e3971, 2008. 